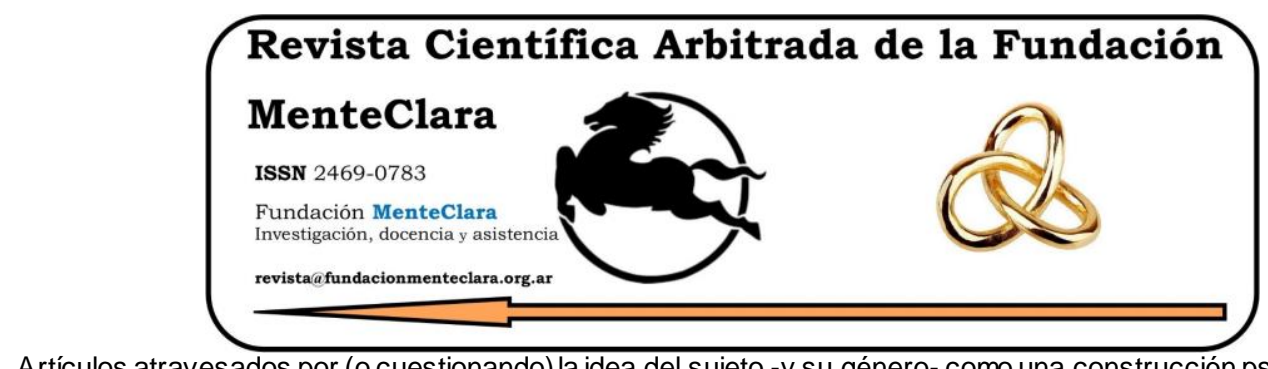

Artículos atravesados por (o cuestionando) la idea del sujeto -y su género- como una construcción psicobiológica de la cultura. Articles driven by (or questioning) the idea of the subject -and their gender- as a cultural psychobiological construction.

Vol. 6 (2021), enero-diciembre ISSN 2469-0783

https: / / datahub.io/ dataset/2021-6-e258

\title{
MAESTROS DE LA ESPIRITUALIDAD CRISTIANA Y RESILIENCIA. UNA APROXIMACIÓN DESDE LA PSICOLOGÍA DE LA SALUD
}

\author{
MASTERS OF CHRISTIAN SPIRITUALITY AND RESILENCE AN APROACH FROM \\ HEALTH PSICHOLOGY
}

Juan Pablo M agnanelli magnanellijuanpablo@gmail.com

Universidad de Flores, Psicología, Psicología de la salud, Argentina.

Cómo citar este artículo / Citation: Magnanelli, J. P. (2021). Maestros de la espiritualidad cristiana y re siliencia. Una a proximación de sde la psicología de la salud. Revista Cientifica Arbitrada de la Fundación MenteClara, Vol. 6 (258). DOI:

https: / /doi.org/ 10.32351/rca.v6.258

Copyright: (C) 2021 RCAFMC. Este artículo de acceso abierto es distribuido bajo los términos de la licencia Creative Commons Attribution 4.0 International License (CC BY 4.0). Recibido: 06/12/2021. Aceptado: 16/12/2021 Publicación online: 20/12/2021

Conflicto de intereses: Ninguno que declarar.

\section{Resumen}

La espiritualidad y la resiliencia son constructos muy importantes de ntro de la psicología de la salud. No obstante, si bien hay muchos estudios que intentan relacionar a la espiritualidad con la resiliencia, creemos que todavía faltan conexiones con respecto a una propuesta especifica de espiritualidad que ayude a una vida en clave de resiliencia; más allá de estar atravesando o no situaciones traumáticas. Es por ello que el presente estudio examinará algunos puntos para la vivencia de una espiritualidad específica: la cristiana. A su vez, observaremos las correspondencias entre las enseñanzas de los maestros de la espiritualidad cristiana y la propuesta de una vida desde la perspectiva salutogénica. Creemos que estas breves líneas pueden ser una aproximación, como un puntapié inicial para desarrollar, en otras investigaciones posteriores, los grandes nexos existentes entre la tradición espiritual cristiana y lo que hoy en dia se desarrolla desde la psicología de la salud para una vida que despliegue todas las potencialidades de la persona humana. 


\begin{abstract}
Spirituality and resilience are very important constructs in health psychology. Although there are many studies that try to relate spirituality with resilience, we believe that there is still a lack of connections with respect to a specific proposal of spirituality that helps to live a life in the key of resilience, beyond being going through traumatic situations or not. In our study we will present some points for the experience of a specific spirituality: the Christian one. At the same time, we will observe the correspondences between the teachings of the masters of Christian spirituality and the proposal of a life from a salutogenic perspective. We believe that these brief lines can be an approximation, as a starting point to develop, in other more profound investigations, the great existing links between the Christian spiritual tradition with what is developed today from the psychology of health for a life that unfolds all the potentialities of the human person.
\end{abstract}

Palabras Claves: Espiritualidad; Resiliencia; Psicología de la Salud; Espiritualidad Ignaciana; Maestros espirituales

Keyw ords: Spirituality; Re silience; Health Psychology; Ignatian Spirituality; Spiritual teachers 


\section{Introducción}

El presente estudio tiene por objeto desarrollar diferentes aportes sobre los conceptos de espiritualidad y resiliencia. En un primer momento vamos a repasar cada uno de los constructos por separado, para después relacionarlos dentro de la perspectiva de la psicología de la salud.

Con respecto a la variable espiritualidad hemos hecho una opción por un tipo concreto de espiritualidad: la espiritualidad cristiana católica. Y más específicamente dentro de la espiritualidad cristiana católica, tomamos los aportes de la espiritualidad ignaciana, proveniente de San Ignacio de Loyola, y la teresiana, siguiendo a Santa Teresa de Ávila.

Asimismo, para el constructo de la resiliencia hemos seleccionado artículos de diferentes autores donde por medio de una serie de estudios empíricos vemos cómo la resiliencia se relaciona con la espiritualidad de manera más general, más allá de la espiritualidad cristiana, pero que sin embargo todos los puntos de relación encuentran conexión con lo que se intenta vivir dentro de la espiritualidad cristiana. En el campo de la psicología de la salud la resiliencia se destaca como una variable de gran importancia para alcanzar el bienestar y adquirir una buena salud mental. Esto lo vemos reflejado en diferentes estudios que recogen Vázquez y Hervás (2009) y Vecina Jiménez (2006). Teniendo en cuenta todos estos factores, la hemos elegido como una variable que puede ser conectada con una forma específica de espiritualidad. Sin más preámbulos, pasamos a desarrollar nuestro trabajo dividido en cuatro puntos: Espiritualidad y religiosidad; Espiritualidad ignaciana: gratuidad, emociones y sentido de la vida; la resiliencia y la psicología de la salud; espiritualidad y resiliencia para una vida más sana. 


\section{Espiritualidad y religiosidad}

Dentro del imaginario común de las personas suele haber una confusión entre lo que significa la espiritualidad y la religiosidad. Resaltamos que en nuestro trabajo hemos hecho una opción dentro del amplio campo de la espiritualidad y escogemos específicamente la espiritualidad católica (ignaciana y teresiana).

Ahora bien, ¿de qué hablamos cuando hablamos de espiritualidad? En primer lugar, es fundamental resaltar las diferencias existentes entre el concepto de espiritualidad y religiosidad. Podriamos decir que la espiritualidad es entendida como una relación o encuentro con algo superior pero más indefinido que nos ayuda en el desarrollo de nuestra capacidad de trascendencia. Por el contrario, la religiosidad la entendemos como un conjunto más definido de normas, ritos, tradiciones, teología, estilo de vida, al cual adherimos mediante el ejercicio espiritual (Del Carmen Fuentes, 2019). En síntesis, la espiritualidad hace referencia a una forma de trascendencia hacia algo más general y la religiosidad apunta a un tipo de espiritualidad concreta y específica. Más allá de estas distinciones, que son importantes tenerlas en cuenta para el desarrollo de nuestro análisis, queremos resaltar el hecho de que tanto la espiritualidad como la religiosidad pueden y deben ayudarnos al desarrollo de un bienestar psicológico (Del Carmen Fuentes, 2019) y, como veremos más adelante, es crucial a su vez para crecer en nuestra capacidad de resiliencia. La apertura espiritual del ser humano está por encima de cualquier religiosidad, esta tendencia nos conecta con lo más profundo del ser humano y si bien nosotros escogimos un tipo de espiritualidad, no queremos decir que lo que planteamos no se pueda encontrar en otras formas de trascendencia (Rodríguez Arenas, 2013). 
La palabra espiritualidad tiene su origen en la tradición cristiana y proviene de diferentes términos en hebreo, latín y griego que apuntan al soplo tanto de aire como de vida (Del Carmen Fuentes, 2019). Podríamos decir que la espiritualidad apunta a la búsqueda de respuestas ante los grandes interrogantes humanos, para poder dar cuenta de la vida con toda la complejidad que encierra. El soplo de vida, según la tradición cristiana, es recibido por parte del Dios creador: "Entonces el Señor Dios modeló al hombre con arcilla del suelo y sopló en su nariz un aliento de vida. Así el hombre se convirtió en un ser viviente" (El libro del Pueblo de Dios, 1990, pág. Gn 2:7). Podemos concluir que la espiritualidad tiene en su origen una búsqueda de aquello que nos vuelve a lo más profundo de nosotros mismos, al sentido último del ser humano donde retornamos a aquella fuente de la vida y donde nos conecta con aquello que está en nuestros comienzos u orígenes. De todas formas, somos conscientes de que la espiritualidad puede no tener esta relación concreta con un ser superior y que tiene su importancia más allá del hecho de una religiosidad específica (Del Carmen Fuentes, 2019). Por esta razón, el constructo espiritualidad tiene una acepción más amplia, amén de que en sus orígenes se encuentre esta relación con el cristianismo.

\section{Espiritualidad ignaciana: Gratuidad, emociones y sentido de la} vida

Luego de hacer estas salvedades entre los equivocos que pueden surgir al hablar de espiritualidad, vamos a focalizarnos en la espiritualidad cristiana. Tomaremos el trabajo de diferentes autores que nos ayudarán a profundizar la espiritualidad desde la alegría y el trabajo de las emociones. 
Dentro de los ejercicios espirituales escritos por San Ignacio de Loyola, encontramos en su experiencia el tema de la gratitud desbordante, él mismo vivió esta gratitud (Boné Pina, 2016). ¿De dónde podemos extraer esta experiencia de gratitud? Desde el convencimiento del regalo y del don que llegamos a experimentar al vivir esta vida y saber que todo es un regalo del cielo (Boné Pina, 2016). La gratitud está presente en los ejercicios espirituales de San Ignacio como un tema central de su espiritualidad. San Ignacio nos invita a abrirnos al agradecimiento y a mantenerlo durante todo el tiempo en que duren los ejercicios como una actitud necesaria para realizar el recorrido espiritual que nos propone. A su vez, desde la psicología positiva se destaca la gratitud como un pilar necesario para ponderar los valores personales y ponerlos a disposición de un completo bienestar de la persona (Boné Pina, 2016). ¿Por qué es importante esta actitud? Porque nos invita a no centrarnos en lo que no tenemos o en lo que podríamos llamar patológico o negativo en nosotros y nos abre potencialmente a lo que podemos llegar a tener, partiendo de una actitud agradecida hacia Dios y los demás (Boné Pina, 2016).

San Ignacio en los ejercicios espirituales nos habla de esta actitud de gratitud al decirnos que, en nuestra relación espiritual con Dios, percibimos que en él no se da un cambio de su actitud hacia mí, su amor siempre se mantiene. Por lo tanto, esta experiencia espiritual que se da en la relación con la divinidad nos abre a la gratitud y a la entrega libre, permitiéndonos crecer en nuestra libertad y salud psíquica (Prieto \& García de Castro, 2016).

Paradójicamente, esta actitud de ir al encuentro de Otro, repercute positivamente en la persona agradecida. Tanto es así que la apertura al agradecimiento ayuda a sobrellevar las situaciones dificiles de una mejor forma que lo pueden llegar a realizar las personas que no son agradecidas (Boné Pina, 2016). Esto no es óbice para quitar el lugar necesario que 
debe tener afrontar los diversos duelosy situaciones traumáticas, incluso llegando hasta una incomprensión con Dios o con la divinidad. Es preciso no saltarnos los procesos, debemos dar espacio al dolor y a la pérdida, pero la propuesta espiritual es hacerlo desde la lógica de la gratuidad (Boné Pina, 2016).

Otro de los puntos es el de las emociones y cómo San Ignacio lo trataba con el nombre de "mociones" buenas o malas (Anchía \& Del Valle Caraballo, 2016). Mediante un proceso natural y complejo las emociones entran en juego ante diferentes situaciones que vivenciamos, dentro del desarrollo filogenético tenían la función de advertirnos o salvarnos de diversos peligros, por eso se disparan mecanismos automáticos que quizás en la vida moderna pueden parecernos molestos. La propuesta de Anchía \& Del Valle Carballo (2016) nos hace ver la importancia de la espiritualidad ignaciana dentro de la comprensión y aceptación del mundo emocional, ya que permite conectar la experiencia personal con nuestro mundo interior dando sentido a la vida desde una perspectiva más amplia y ordenadora de la misma existencia. Dicho de otro modo, la espiritualidad nos ayuda a unir nuestra vida interior y la experiencia personal dentro de un proyecto que nos trasciende y en el que estamos inmersos.

Desde la experiencia espiritual cristiana muchas de las cosas que se nos plantea vivir desde la Psicología Positiva, suenan a terreno conocido, ya que la espiritualidad hace hincapié en una vida de autoconocimiento, de búsqueda de sentido, de acción de gracias, de perdón y nos abre a la experiencia del amor incondicional (Ruiz, 2016). Creemos que ambos terrenos se pueden enriquecer mutuamente desde los aportes de cada área en pro del bienestar integral de la persona. Muchas de las cosas que la Psicología Positiva nos plantea y las diferentes terapias que se ofrecen para alcanzar dichos objetivos, podemos afirmar que ya estaban 
presentes en los grandes maestros de espiritualidad cristiana (Ruiz, 2016).

Con respecto al tema del perdón, constatamos que realmente se da una trasformación integral de la persona, incluso fisicamente, pero más importante aún es lo que se registra en el cambio de la percepción de las cosas, la forma de comportarse y los pensamientos. Todo lo cual repercute en la posibilidad de tener una vida plena (Prieto \& García de Castro, 2016). Siguiendo esta línea, podemos afirmar que el perdón libera tanto al perdonado como al que perdona, esta fuerza del perdón nos abre a la resiliencia, la plenitud de nuestra vida y la de los que nos rodean (Rodríguez Arenas, 2013).

En efecto, Ruiz (2016) nos propone doce puntos que nos pueden ser de provecho para llevar una vida con sentido y que también están presentes en la tradición espiritual ignaciana. Vamos a escoger cinco puntos para nuestro desarrollo. En primer lugar, encontramos la propuesta de "vivir el presente", detenernos en lo que estamos haciendo, sintiendo, viviendo. Buscar espacio para hacer aquello que nos gusta. En segundo lugar, escogimos "dar sentido al dolor y la muerte", para ello es importante aprender a dejarse ayudar, dar lugar al dolor, narrar o incluso escribir lo que nos pasa. En muchas situaciones el dolor nos hace estar fuera de nosotros mismos, nos sentimos desconocidos y es preciso realizar un proceso de vuelta a casa para aprender a entrar en lo más profundo de nosotros (Bofill \& Mollá, 2016).

En tercer lugar "aprender a mirar afuera de uno mismo", es necesario no quedarnos en ese continuo rumiar que nos puede llegar a perjudicar, dar ese salto hacia el exterior puede ayudarnos a cortar con pensamientos negativos. En este sentido cabe destacar que el Dios de la espiritualidad cristiana, el Dios de Jesús, nos compromete en una 
búsqueda de justicia y solidaridad en medio del mundo (Domínguez Morano, 2009), de esta forma nos ayuda a salir de nosotros mismos para mirar el dolor del otro. Por otra parte, nos propone escoger un estilo de vida que nos permita "ser optimista", a pesar de las dificultades hay que aprender a proyectar vivencias que nos ayuden a cumplir los objetivos de nuestra vida y poner empeño para alcanzarlos. Por último, “practicar la amabilidad", teniendo una actitud afable con los que nos rodean, generar un buen clima a nuestro alrededor, cuidando nuestras palabras y buscando las cosas positivas del otro y del ambiente (Ruiz, 2016).

\section{La resiliencia y la Psicologia de la Salud}

Luego de definir la variable espiritualidad y de explorar diferentes relaciones entre la espiritualidad cristiana y la psicología, pasamos a abordar la variable resiliencia y ver su relación con la búsqueda de sentido para una vivencia óptima de la salud mental.

Cuando hablamos de resiliencia tenemos que tener en cuenta en un primer momento que en su origen es un término prestado de otro campo, el de la física. Dicho término se refiere a la capacidad que tienen los materiales para resistir o recuperarse, luego de ser sometidos a diferentes fuerzas (García del Castillo Rodríguez, García del Castillo López, LópezSánchez, \& Dias, 2016). Podemos hacer así una analogía con las diferentes experiencias que pasamos a lo largo de la vida y que vamos superando y nos van transformando o cambiando. Las diferentes definiciones de resiliencia nos hablan de adaptación, habilidad para afrontar las dificultades, interacción de factores internos y externos, y de resiliencia como proceso (García del Castillo Rodríguez, García del Castillo López, López-Sánchez, \& Dias, 2016). Por la experiencia, podemos afirmar que todos atravesamos diferentes momentos de crisis. 
La resiliencia nos ayuda a superar esas situaciones y nos hace sacar experiencias positivas que nos permiten crecer en otros ámbitos de la vida. Es significativo observar cómo las personas que tienen capacidad de resiliencia logran mantener un mayor nivel de salud en general, más allá de estar atravesando o no situaciones traumáticas (García del Castillo Rodríguez, García del Castillo López, López-Sánchez, \& Dias, 2016).

Si bien hay ciertos acuerdos con respecto a la definición de resiliencia también tenemos que decir que en diferentes autores encontramos variantes que influyen de forma no menor en el significado de nuestra variable. Algunos insisten en definirla como un rasgo de la persona, otros apuntan a verla más como un proceso. Quizás este segundo grupo de definiciones es el que más nos interesa a nosotros ya que nos permite tomar la resiliencia como un constructo que todos podemos trabajar más allá de que algunas personas tengan mayor inclinación a practicarla (Ortunio \& Guevara, 2016). Otra de las cosas interesantes con respecto a la resiliencia es la posibilidad de que ésta sea no solamente vista para afrontar las grandes dificultades de la vida sino como una ayuda para llevar adelante las situaciones cotidianas y reducir el estrés que producen los problemas cotidianos (Ortunio \& Guevara, 2016).

A su vez, aquellos que trabajan en la Psicologia positiva toman esta variable como una bandera propia de su movimiento, pero hay que decir que pertenece a todos los campos de la psicología (Ortunio \& Guevara, 2016). Nosotros, justamente la estamos relacionando con la espiritualidad, pero no para equipararla, sino para fortalecerla $y$ potenciarla dentro de una perspectiva más amplia. 
Por último, más allá de los diferentes tintes que podemos encontrar con respecto a la definición de resiliencia, también encontramos diferentes formas de medirla. Es preciso tener en cuenta que nuestro constructo mayormente fue tenido en cuenta para el análisis de niños o adolescentes que han atravesado diferentes procesos traumáticos, faltan estudios en la población adulta y otro de los inconvenientes es la relación constante de la resiliencia con lo psicopatológico (Ortunio \& Guevara, 2016). Por este motivo, queremos destacar la importancia de no asociarla únicamente a lo patológico o problemático, sino en la posibilidad de verla como un desarrollo deseable para todas las personas en cualquier situación de la vida que se encuentren y lograr así, acrecentar los niveles de bienestar integral. Para lograr esto Ortunio y Guevara (2016) nos hablan de los diferentes estudios realizados en situaciones estresantes cotidianas por estudiantes y la capacidad de resiliencia para afrontar esos momentos. Hay una interrelación entre emociones positivas, capacidad de afrontar el estrés y resiliencia que nos invita a potenciar el cultivo de estas capacidades en el desarrollo del proceso de crecimiento humano.

En un estudio de investigación de diversos autores que realiza Cala (2020) sobre la resiliencia y su impacto en la salud de las personas, se puede observar desde las diferentes perspectivas una conclusión común que nos habla de la importancia de la resiliencia para la salud tanto física como mental y la ayuda que realiza para el bienestar completo de la persona. A su vez, Cala (2020) destaca la importancia de la resiliencia con la salud y nos muestra cómo se pueden conjugar diferentes variables en torno a una vida en clave de resiliencia entre las cuales se encuentra una que nosotros venimos trabajando: la religiosidad. 
Luego de este repaso por las variables espiritualidad y resiliencia vamos a explorar de qué forma podemos llegar a combinar nuestras variables dentro del estudio de la psicología de la salud.

\section{Espiritualidad y Resiliencia para una vida más sana}

La espiritualidad desde la perspectiva que estamos trabajando es aquella que nos permite autotrascendernos e ir más allá de nosotros mismos, a su vez, esta actitud interior nos ayuda a encontrar un sentido en situaciones traumáticas. Según Rodríguez Fernández (2016) algo para destacar es que la mayoría de las personas (91\%) que atraviesan situaciones traumáticas no registran problemas mentales, por lo tanto, es posible atravesary superar esas situaciones sin padecer traumas por ello. Continúa Rodríguez Fernández (2016) diciéndonos que las personas que pudieron encontrarse con su mundo interior y autotrascenderse por medio de aquello que nosotros hemos denominado espiritualidad, son las personas que registran un crecimiento y aprendizaje mayores luego de la situación traumática. Más aún, las personas que ya poseían este recurso espiritual antes del trauma, son aquellas que pudieron sobreponerse con mayor rapidez. De todas formas, no todo es color de rosa en este viaje hacia al interior, ya que podemos encontrarnos con algunas sombras que nos gustaría esquivar. Pero por más paradójico que pueda parecernos, este recorrido nos ayuda a encontrarnos con lo más profundo de nosotros mismos, y por ende a encontrar mayor libertad y autenticidad en nuestra vida. Quizás la situación traumática es la puerta que nos permite entrar a una vida plena de sentido. Contamos con los testimonios de muchos místicos de la tradición cristiana y con algunas personas reconocidas dentro del campo de la psicología que han hecho esta experiencia (Rodríguez Fernández, 2016). 
No podía faltar en nuestro estudio el aporte de Viktor Frank1. Podemos llegar a ver cómo la espiritualidad y la resiliencia se encuentran interconectadas en la experiencia de Viktor Frankl. Rodríguez Fernández (2016), nos muestra diez puntos que resumen la experiencia de Viktor Frankl y cómo ésta se conecta con nuestras dos variables. Nosotros tomaremos a modo de ilustración algunos de ellos: El sentido de sufrimiento, la atención al presente y cultivar la autotrascendencia. En relación al sentido del sufrimiento, vemos cómo el sufrimiento puede ser una oportunidad para poner en marcha una serie de recursos desconocidos. Es necesaria una actitud valiente para atreverse a afrontar el sufrimiento. Por otro lado, descubrimos en Frankl la atención al presente. Esto es algo que más arriba desarrollamos al hablar de San Ignacio. Esta atención es para Frankl la que nos permite encontrar en nuestro día a día lo que estamos atravesando y abrirnos al contenido de nuestra existencia en general. Por último, al hablar de cultivar la autotrascendenciadecimos que esta capacidad es la que nos permite salir de nuestro reducido punto de vista y mirar el sufrimiento de los demás. Esta actitud nos aleja del autoaislamiento egoísta (Rodríguez Fernández, 2016).

Continuando con nuestra línea de trabajo vemos cómo Irurzun et al. (2017), se proponen investigar los diferentes estudios que relacionan la espiritualidad y la resiliencia. Si bien quedan muchos trabajos para seguir ahondando en el tema, queda claro que hay una estrecha relación entre nuestras variables. Destacamos la importancia de la forma en que la persona vive su espiritualidad y su contacto con el ambiente religioso, ya que, si no es positivo y si su vivencia espiritual está mal orientada, puede traerle más dificultades que beneficios (Irurzun, Mezzadra, \& Preuss, 2017). De todas formas, en diferentes análisis de poblaciones que vivenciaron todo tipo de situaciones dolorosas, se muestra cómo la 
espiritualidad ayuda a salir de esos momentos y abre la posibilidad de tener un mayor bienestar de las personas. Asimismo, se constata la necesidad de un mayor grado de estudios empíricos en relación a este tema (Irurzun, Mezzadra, \& Preuss, 2017).

En otro trabajo de investigación Irurzun y Yaccarini (2019) relacionan también ambos constructos y se suma la variable "propósito de vida". Podemos observar cómo, si bien ya muchos autores ven la relación existente entre espiritualidad y resiliencia, agregar un propósito de vida a la persona ayuda a tener una visión más amplia hacia el futuro y a desplegar una serie de recursos que lo alejan del pensamiento del dolor presente y lo invitan a ver su vida con otra mirada más abarcadora, valorando y poniendo en relación diferentes situaciones que le permiten afrontar la vida con un impulso diferente que los moviliza. El sentido de la vida motiva internamente a la persona para que pueda vivir su espiritualidad y por ende tenga una mayor capacidad de resiliencia, de ahí la interrelación de estas tres variables. Sin embargo, la relación entre sentido de vida y resiliencia todavía debe ser más estudiada, ya que encontramos más estudios que apuntan a la conexión entre resiliencia y espiritualidad (Irurzun \& Yaccarini, 2019).

\section{Conclusión}

Luego de nuestro recorrido bibliográfico pudimos observar cómo la espiritualidad y la resiliencia pueden interconectarse y enriquecerse mutuamente. A su vez, nuestra opción por la espiritualidad católica nos dio la posibilidad de contrastar la tradición cristiana y diferentes maestros de la vida espiritual con una vivencia práctica de la relación de ambos constructos. Pudimos observar que sus enseñanzas están hoy en día abiertas para trabajar como una forma concreta de crecer en la 
espiritualidad y poder así aumentar nuestra capacidad de resiliencia. Antes de finalizar nuestro recorrido cabe realizar una aclaración. Si bien intentamos enriquecer la mirada con los aportes de la psicología y de la espiritualidad cristiana también hay que decir que es fundamental mantener los límites específicos entre cada campo, de esta forma podremos lograr un diálogo fecundo que sin confundir pueda enriquecerse con ambos aportes (Dominguez Morano, 2006).

Queda pendiente un desarrollo más amplio que conecte estas dos variables con el sentido de la vida, pero pudimos perfilar que ya hay bastantes indicios que nos permiten hacer una estrecha relación entre los tres constructos. Creemos que los grandes maestros de espiritualidad católica son lo que nos impulsan desde hace siglos en esa dirección, por esta razón nos gustaría que se nos permita concluir nuestra reflexión con un poema de una mujer que supo conjugar ambas variables en su vida personal y nos deja un legado para animarnos a avanzar en este mismo sentido espiritual y resiliente. Que sea ella la que nos hable y que la fuerza de sus palabras nos mueva a avanzar en esta senda propuesta:

"Nada te turbe

Nada te espante

Todo se pasa

Dios no se muda

La paciencia

Todo lo alcanza

Quien a Dios tiene

Nada le falta

Solo Dios basta"1

(Teresa de Jesús, 1967, pág. 511).

\footnotetext{
${ }_{1}^{1}$ Permítase nos como hizo Rodriguez Fernández (2016), recomendar a los lectores la escucha de este poema interpretado musicalmente por Taizé: https://www.youtube.com/watch?v=go1-BoDD7CI
} 


\section{Referencias}

Anchía, R. J., \& del Valle Caraballo, C. (2016). Emociones y mociones: aportaciones desde la terapia focalizada de la emoción. Manresa: Revista de Espiritualidad Ignaciana., 88(349), 341-352.

Bofill, E. \&. (2016). Interpretar, discernir, acoger: del síntoma a la con-moción. . Manresa: Revista de Espiritualidad Ignaciana, 88(349), 353-362.

Boné Pina, I. (2016). Psicología de la gratitud y ejercicios espirituales. . Manresa: Revista de Espiritualidad Ignaciana., 88(349), 385-398.

Cala, M. L. (2020). La salud desde la perspectiva de la resiliencia. Archivos de Medicina (Manizales)., 20(1), 203-216. doi:https://doi.org/10.30554/archmed.20.1.3600.2020

Del Carmen Fuentes, L. (2019). La Religiosidad y la Espiritualidad ¿'Son conceptos teóricos independientes? Revista de Psicología., 14(28), 109-119.

Domínguez Morano, C. (2006). Ignacio de Loyola a la luz del psicoanálisis. Proyección: Teología y mundo actual., 53(222), 25-56.

Domínguez Morano, C. (2009). Teología y psicoanálisis de la experiencia religiosa. Revista Iberoamericana de Teologia., 5(9), 45-69.

El libro del Pueblo de Dios. (1990). Obtenido de https://www.vatican.va/archive /ESL0506/__P3.HTM

Finez-Silva, M. J., Morán-Astorga, C., \& Urchaga-Litago, J. D. (2019). Resiliencia psicológica a través de la edad y el sexo. International Journal of Developmental and Educational Psychology., 4(1), 85-94. doi:https://doi.org/10.17060/ijodaep.2019.n1.v4.1513

García del Castillo Rodríguez, J. A., García del Castillo López, Á., López-Sánchez, C., \& Dias, P. C. (2016). Conceptualización teórica de la resiliencia psicosocial y su relación con la salud. Salud y drogas, 16(1), 59-68.

Irurzun, J., \& Yaccarini, C. (2019). Resiliencia, espiritualidad y propósito de vida. Una revisión de1 estado del arte. Psocial, 4(2), 58-66.

Irurzun, J., Mezzadra, J., \& Preuss, M. (2017). Re siliencia y espiritualidad. Aportes para su estudio desde una perspectiva psicológica. Revista Científica Arbitrada de la Fundación MenteClara., 2(2), 205-216. doi:https://doi.org/10.32351/rca.v2.2.34

Mendoza, R. (2016). Resiliencia y calidad de vida. La Psicología Educacional en diálogo con otras disciplinas. Universum (Talca), 31(2), 247-250.

Ortunio, M. S., \& Guevara, H. (2016). Aproximación teórica al constructo resiliencia. Comunidad y Salud, 14(2), 96-105.

Prieto, M. \&. (2016). Psicología del perdón y Ejercicios Espirituales. . Manresa: Revista de Espiritualidad Ignaciana., 88(349), 317-328.

Rodríguez Arenas, M. S. (2013). La resiliencia como vivencia de1 Reino de Dios. Lectura teológica de la resiliencia. Pontificia Universidad Javeriana.

Rodríguez Fernández, M. I. (2016). Espiritualidad, resiliencia y creci miento postraumático. NOUS, Revista de Logoterapia y Análisis Existencial., (19), 21-32.

Ruiz, A. S. (2016). Fortalezas personales y vida con sentido: aportaciones para el acompañamiento. Manresa: Revista de Espiritualidad Ignaciana., 88(349), 329-340.

Teresa de Jesús, S. (1967). Obras Completas. Biblioteca de autores cristianos. 
Vázquez, C., \& Hervás, G. (2009). Psicología positiva aplicada. Desclée de Brouwer.

Vecina Jiménez, M. (2006). Psicología Positiva: Emociones positivas. Papeles del Psicólogo. 27(1), 917 . 